\title{
Isolation and characterization of the ATP-binding cassette (ABC) transporter system genes from loofah witches' broom phytoplasma
}

\author{
CHUN-LIN HUANG ${ }^{\dagger} \&$ KUO-CHIEH HO \\ Department of Life Science, Institute of Plant Biology, National Taiwan University, Taipei, Taiwan
}

(Received 26 Fuly 2006)

\begin{abstract}
A clone containing a 3903 bp EcoRI-restriction fragment was obtained from a $\lambda_{\text {ZAP }}$ genomic library of loofah witches' broom (LfWB) phytoplasma by plaque hybridization using a PCR fragment as a probe. Sequence analysis revealed that this fragment contained three open reading frames (ORFs). The deduced amino acid sequences of ORF 1 and ORF 2 showed a high homology with the ATP-binding proteins of the ABC transporter system genes of prokaryotes and eukaryotes, and encoded proteins with a molecular mass of 36 and $30 \mathrm{kDa}$, respectively. Based on amino acid sequence similarity, secondary structure, hydrophilicity and a signal peptide sequence at the N-terminus, we predicted that ORF 3 might encode a specific solutebinding prolipoprotein of the ABC transporter system with a molecular mass of $62 \mathrm{kDa}$. The cleavage site of this prolipoprotein signal peptide was similar to those of gram-positive bacteria. In addition to nutrient uptake, ABC transporter systems of bacteria also play a role in signal transduction, drug-resistance and perhaps virulence. The possible implications of the system to the survival and the pathogenesis of phytoplasma were discussed.
\end{abstract}

Keywords: Loofah witches' broom (LfWB), phytoplasma, ATP-binding cassette ( $A B C)$ transporter system, open reading frames

Database accession number: AF086618 (P36), AF086619 (P30), AF086620 (P62)

\section{Introduction}

Phytoplasmas, a member of mollicutes, are the causal agents of more than 200 diseases of higher plants (Kirkpatrick 1989; McCoy et al. 1989). They have remained uncultured in vitro and their inability to grow in culture medium has severely hindered their studies (Lee and Davis 1986). As a result, phytoplasmas are the most poorly characterized groups of plant pathogens. Although phytoplasmas' host ranges (plant and insect) and symptomatology (phenotype differences) are similar, their genotype and genome sizes (600$1200 \mathrm{kDa}$ ) are diverse (Razin 1992).

Phytoplasmas were known previously as mycoplasmalike organisms (MLOs). However, phylogenetic relationships based on the sequences of the full-length $16 \mathrm{~S}$ rRNA, ribosomal protein genes and the 16/23S spacer regions revealed that phytoplasmas form a large discrete monophyletic clade, paraphyletic to the
Acholeplasma species, within the Anaeroplasma clade rather than Mycoplasma (Tully 1993; Gundersen et al. 1994; Sear and Kirpatrick 1994; Ho et al. 2001). Recently, phytoplasma can be detected, identified and classified clearly by molecular methods. While these phylogenetic advances continue, research has focus on the pathogenicity genes. These result in the novel approaches to achieve effective control of phytoplasmal diseases (Kirkpatrick and Smart 1995; Lee et al. 2000; Wagner et al. 2001) and the completion of two phytoplasma genome sequences (Oshima et al. 2004; Bai et al. 2006).

The ATP-binding cassette (ABC) superfamily is one of the largest protein families found in living systems, with several hundred different genes identified to date in organisms ranging from bacteria to man. Typically, ABC transporters utilize the energy of ATP to pump substrates across the membrane against

Correspondence: K. -C. Ho, Department of Life Science, National Taiwan University, 1 Roosevelt Road, Sec. 4, Taipei 106, Taiwan. Tel: 8862 33662508. Fax: 8862 23637274. E-mail: kch@ntu.edu.tw

†Department of Botany, National Museum of Natural Science, Taichung, Taiwan. E-mail: wagtail@mail.nmns.edu.tw. 
a concentration gradient. The common protein components of the ABC-type uptake systems include one or two transmembrane proteins which usually span the membrane five to six times each, one or two peripheral membrane ATP-binding proteins in the cytoplasmic side and a high-affinity extra-membrane solute-binding protein (Higgins 1992; Locher 2004; Khwaja et al. 2005). In gram-negative bacteria, the ligand-specific binding protein is soluble and periplasmic. In gram-positive bacteria, the solublebinding protein is also extracellular and anchors to the membrane via an $\mathrm{N}$-terminal hydrophobic lipid extension. The transmembrane protein components are believed to form solute-specific channels; the peripheral membrane ATP-binding proteins energize the systems and sometimes serve regulatory roles, and the ligand-binding proteins confer specificity and high affinity for the substrates to the transporter systems (Ames 1986; Shuman 1987; Tam and Saier 1993; Monnet 2003). In eukaryotic cells, ABC transporters have been predominantly found in the plasma membrane where they catalyze the efflux of various compounds out of the cell. Other ABC proteins are located in intracellular organelles such as peroxisomes, mitochondria, the endoplasmic reticulum and vacuoles; some of these proteins mediate the compartmentation of compounds into the organelles (Davies and Coleman 2000; Young et al. 2001).

The ABC transporters can transport a remarkable variety of substrates, including ions, carbohydrates, lipids, antibiotics, anti-cancer drugs, pigment molecules and even large peptides. They participate in many biological functions, e.g. phosphate regulation in Escherichia coli (Saier 1993), chemical signaling between Agrobacterium tumefaciens and its plant host (Ankenbauer and Nester 1990; Cangelosi et al. 1990), control of Bacillus subtilis sporulation (Perego et al. 1991; Koide and Hoch 1994), transport of sex pheromones in yeasts (McGrath and Varshavsky 1989), and chloride via the cyctic fibrosis transport regulator protein in mammals (Riordan et al. 1989; Tata et al. 1991). The vast majority of studies on ABC transporters have been driven by their diverse importance.

In this communication, we reported the cloning and characterization of a DNA fragment containing the ABC transporter system genes from loofah witches' broom (LfWB) phytoplasma. The possible implications of the system to the survival and the pathogenesis of phytoplasma were also discussed.

\section{Materials and methods}

\section{Bacterium and plant}

The LfWB phytoplamas were maintained by graft inoculation in periwinkles (Chen and Ho 1997). The original diseased plant was provided by Dr H.-J.
Su, Professor of Department of Plant Pathology and Microbiology, National Taiwan University, Taiwan.

\section{Phytoplasma genomic library construction and screening}

The following methods, including healthy or diseased plant DNA extraction, phytoplasma DNA purification and phytoplasma genomic library construction were done as previously described (Ho et al. 2001).

The library was screened by plaque hybridization using a ${ }^{32}$ P-lablled PCR fragment amplified on the LfWB phytoplasma DNA with primers RN1 (5'-CGCTTAGAGTTTAGGTGA-3') and RN2 (5'-CCAACCAAAGCTTC-3'), synthesized according to the DNA sequence of ribonuclease III of Mycoplasma genitalium (Fraser et al. 1995). The positive plaque areas were selected and rescreened until a single, isolated plaque could be picked up.

\section{Southern blot analysis}

Phytoplasma DNA ( $3 \mu \mathrm{g})$ or plant DNA $(10 \mu \mathrm{g})$ were digested with different restriction enzymes and subjected to Southern blot analysis performed at $42^{\circ} \mathrm{C}$ in the presence of $50 \%$ formamide and $0.1 \%$ SDS using the ${ }^{32} \mathrm{P}$-lablled DNA probe. The filter was washed with $0.1 \times$ SSC $(1 \times$ SSC: $150 \mathrm{mM} \mathrm{NaCl}$, $15 \mathrm{mM}$ sodium citrate) containing $0.1 \% \mathrm{SDS}$ at $50^{\circ} \mathrm{C}$. The hybridized bands were detected by exposing the filter to a PhosphoImager screen (PhosphoImager 425; Molecular Dynamics).

\section{Sequence determination and analysis}

For DNA sequencing, the recombinant phage was converted into a phagemid by in vivo excision according to manufacturer's instructions (Stratagene). The sequence of DNA insert in the recombinant phagemid was determined by a DNA automated sequencer (ABI Prism Model 377, v.30; Applied Biosystems) using a step-by-step procedure in which synthetic primers for forward sequencing were designed from previously released sequences. DNA sequence analysis was performed using DNAStar software (DNASTAR). The deduced amino acid sequences were analyzed through the BLAST of National Center for Biotechnology Information sever (http://www.ncbi.nlm.nih.gov/BLAST), and submitted to the 3D-PSSM fold recognition server (http://www.sbg.bio.ic.ac.uk/ 3dpssm/) for prediction of secondary structure if it is necessary (Kelley et al. 2000). The alignment of multiple sequences was performed by the program Clustalx ver 1.8 (Thompson et al. 1997). 


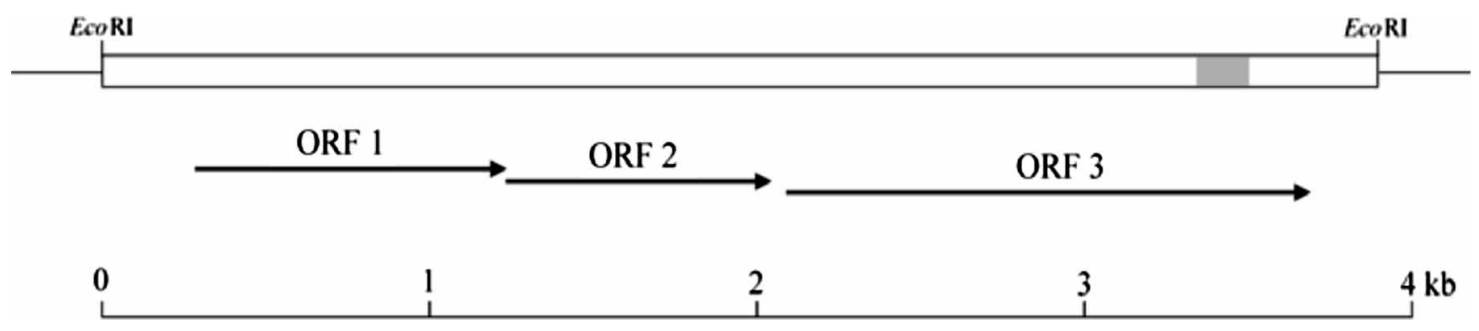

Figure 1. Schematic representation of the $3903 \mathrm{bp}$ cloned-DNA fragment. Arrows indicate the lengths and orientations of the three ORFs. The gray area is the PCR fragment RN-P used as a probe for the hybridization analyses.

\section{Results and discussion}

Nucleotide sequence analysis of the cloned DNA fragment

A 170-bp PCR product, named RN-P was amplified on the phytoplasma DNA. Three clones were obtained using RN-P as a probe to screen an $E c o$ R I-genomic library of LfWB phytoplasma constructed on $\lambda_{\text {ZAP }}$ vector. The DNA sequences indicated that these clones contained the same DNA insert of $3903 \mathrm{bp}$.

The nucleotide sequence of the DNA insert was composed of $76.53 \%$ A $+\mathrm{T}$ which corresponded with the low $\mathrm{G}+\mathrm{C}$ content of phytoplasma genome. No strong homology was found by comparing the nucleotide sequence with those deposited in GenBank. However, the deduced amino acid sequence revealed that this 3903bp DNA insert contained three open reading frames (ORFs) (Figure 1). The ORF 1, from nucleotide 290 to 1237 , encoded a protein of $36 \mathrm{kDa}$ (designed as protein P36). The ORF 2, from nucleotide 1237 to 2031, had one base overlapping with ORF 1 and encoded a protein of $30 \mathrm{kDa}$ (designed as protein P30). The ORF 3, from nucleotide 2090 to 3688 and separated by 60 nucleotides from ORF 2, encoded a protein of $62 \mathrm{kDa}$ (designed as protein P62). All three ORFs had an ATG as a start codon, which was preceded by a ribosome binding site. There was a putative -10 sequence and a putative -35 sequence upstream from the ORF 1, however, no obvious sequence for a rho-dependent or -independent termination site was found downstream from the ORF 3 (Figure 2). Transcription termination at indiscrete sites has been reported for several protein-encoding genes and ribosomal genes of archaeobacteria, where transcription stops within or near a T-rich sequence (Ho et al. 2001 and references therein). It was possible that the transcription of this $\mathrm{ABC}$ transporter operon terminated in one of several pyrimidine-rich regions downstream from the ORF 3, as found in ribosomal gene of the same organism (Ho et al. 2001).

The primers $\mathrm{RN} 1$ and $\mathrm{RN} 2$ were originally designed from mycoplasma sequence to screen the library for the gene encoding ribonuclease III of LfWB phytoplasma. Instead, the DNA insert containing
ABC transporter genes was obtained due to the existence of partial sequence homologies.

P36 and P30 have homology with ATP-binding proteins

The deduced amino acid sequences were used to search for protein homologies by BLAST from protein sequence database. Proteins P36 and P30 displayed a significant homology with several ATP-binding proteins. The features of ATP-binding proteins including (1) Walker A motif, a glycine-rich loop involved in ATP binding; (2) the ABC signature motif or C motif; (3) the Walker B motif that is associated with many nucleotide-binding proteins; and (4) a switch region characterized by an invariant histidine residue or $\mathrm{H}$ motif, which was suggested to be involved in ATP hydrolysis (Higgins 1992; Zhao et al. 2004) were found in P36 and P30. P30 has shorter sequence length truncating at C-terminus, as it's homolog, DppD of onion yellow (OY) and aster yellows witches'-broom (AYWB) phytoplasmas (Figure 3). Many truncated proteins have been found in the OY and AYWB phytoplasma genomes (Bai et al. 2006). This might be the unique feature of phytoplasma associated with their small genome size.

Hydropathic analysis by the method of Kyte and Doolittle (1982) revealed that P36 and P30 were fairly hydrophilic peripheral membrane proteins as the ATP binding proteins of complex bacterial transporter system (Figure 4A, B). P36 and P30 could form a common engine, which binds and hydrolyzes ATP, and energizes unidirectional substrate transport as the common architecture of $\mathrm{ABC}$ transporters. This engine is attached to a specialized translocon composed of two transmembrane proteins.

\section{P62 is a hypothetical extracellular solute-binding lipoprotein}

The BLAST database search result showed that the P62 was most similar to the solute-binding protein DppA of the OY and AYWB phytoplasma (Bai et al. 2006), but with only $54 \%$ similarity and $48 \%$ similarity, respectively. The DppA of ABC dipeptide transporter system belongs to the extracellular solutebinding lipoprotein cluster 5 . The proteins of cluster 5 
gaat tcaaatctttttcccaaaataggt tattttaagttaaatattttatttgggattta 60

tttagcaattttatttttgattgaaataattaaaagcagttctgtaattgcttttatttt 120

$-35$

ttttaaaaaaacatttgactattacaaaaaaaatgtgttataattttttaaacagttaat 180 $-10$

aaatatttaa tactgttttaataaatattttgcttttagttgtgttttcgactgagata 240

$$
\text { SD P36 }
$$

ttatttaaatcatgat tacata taaaaa tataagaaagtgtaa taatcgatgttgaaaga 300 M L K K $\quad$ D

tcggaatgaaaaa tatcaaattttaatcgagtgcaagaatttatctaaatttttttttag 360

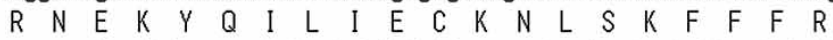

aaagaaattatttgcgaaatctagtatttttttaaaagctaatgatgatattagtttatc 420

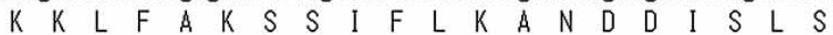

tattcataaagggcaaact ttagccgtagtaggt ggt tcaggaaaatctactttaggaca 480

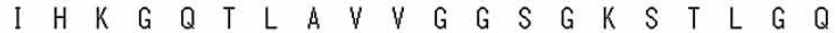

aactttgttgcaattagaaaaatctact tctggaaaagttatt tat tataaagaacagca 540

$\begin{array}{llllllllllllllllllll}T & L & L & Q & L & E & K & S & T & S & G & K & V & I & Y & Y & K & E & Q & Q\end{array}$

atctattaatttaagcgaactatctactaaagatttacaaattattttccaaaatcctta 600

$\begin{array}{llllllllllllllllllll}S & I & N & L & S & E & L & S & T & K & D & L & Q & I & I & F & Q & N & P & Y\end{array}$

tctatctttgaatcctaaaatt ttaatatcagatatcattggcgaaggtcttttaataca 660

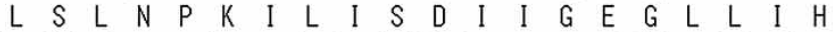

taatttagtaaaaagtaaaaatgatcctctttataaacaaaaattttagatatcatgaa 720

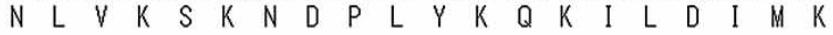

aaaatgcggcgtaaaagaggagttatataatcgttctcttgctcaattatcgggtggcga 780

K C C G V V K $\quad E \quad E \quad L \quad Y \quad N \quad R \quad S \quad L \quad \& \quad Q \quad L \quad S \quad G \quad G \quad E$

aagacaaagaatcgctattgcaagaactttaattatagaacctaaatttattgtttgtga 840

$\begin{array}{llllllllllllllllllll}R & Q & R & I & A & I & A & R & T & L & I & I & E & P & K & F & I & V & C & D\end{array}$

tgaaattgtttctcttttagatgtcacgatacaaaaacaaattttggaattattgaaaca 900

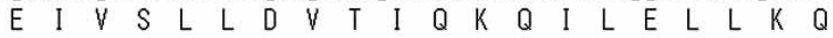

attaaagaaaaat tatgaattaact ttattgtttattactcatgatttaggggttgctaa 960

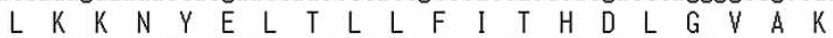

atatttaagcgatcaaattgcaataatgtatttaggtaaattagtagaattaggcccaac 1020

$\begin{array}{llllllllllllllllllll}Y & L & S & D & Q & I & A & I & M & Y & L & G & K & L & V & E & L & G & P & T\end{array}$

agaaaaaatttttacaaaccctcaacatccttatacaattgaattgttaaattctatccc 1080

$\begin{array}{llllllllllllllllllll}E & K & I & F & T & N & P & Q & H & P & Y & T & I & E & L & L & N & S & I & P\end{array}$

taaattgattgaa tatggtatatcaagcaaaaaatacattgctaaatatgaaaat tct tc 1140

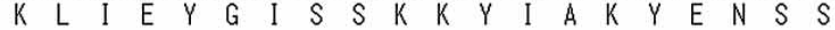

ttatgattttttatacaataagaaaaaagaagataaggattggcatcaagtttctccaaa 1200

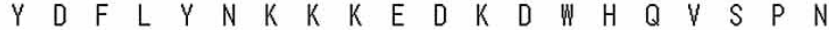

$$
\text { SD P30 }
$$

tcactttattttatgtactaaaaaaagaggctat taatgaaaattt tagaagtaaataat 1260

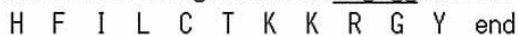

$M$ K I L E V N N

ttacatacttattttgaaactgaaaaaggtttagttaaagctgtacaaggtatttctttt 1320

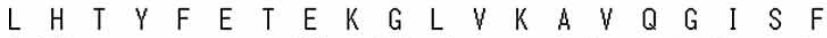
tatttagaacaaggaaaaactttgggtttagtcggagaatctggaagtggtaaaagtcaa 1380 $Y \quad L \quad E \quad Q \quad G \quad K \quad T \quad L \quad G \quad L \quad V \quad G \quad E \quad S \quad G \quad S \quad G \quad K \quad S \quad Q$ acagctaattctattttgaaat tatttgaaaaaacacaaaaaattcatcaaggggaaatt 1440

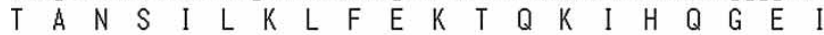
atttttaataatcaagttatttctgattttaatgaaaaaaaaatgcaaaagattcgtggt 1500

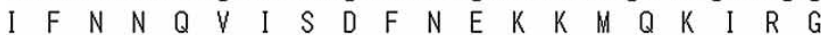
aaagagatatccatgatttttcaagatcctattactagtttaaaccccatgtttaaaata 1560

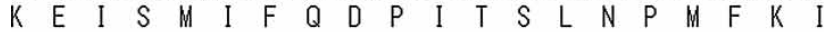
aaagatcaaattatggaaattttatttattcatcaagaaatcacttataaagaagcttat 1620 $\begin{array}{llllllllllllllllllll}K & D & Q & I & M & E & I & L & F & I & H & Q & E & I & T & Y & K & E & A & Y\end{array}$ gaaaaagctttaaatatgt taaaaaaaactaaaattcataattgtgaacaaattatgaat 1680

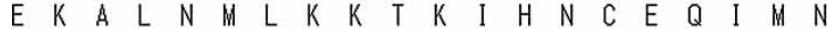
tgttacccttatcaat tatctggcggaatgtgccaaagagtaatgatcgcaatagcttta 1740 $\begin{array}{lllllllllllllllllllll}C & Y & P & Y & Q & L & S & G & G & M & C & Q & R & V & M & I & A & I & A & L\end{array}$ atttgtcaacctaaat tat taatcgtcgatgaagctactatagctt tagatgttatcgtt 1800 $\begin{array}{llllllllllllllllllll}I & C & Q & P & K & L & L & I & V & D & E & A & T & I & A & L & D & V & I & V\end{array}$ caaaaagaaattttgaatttgattaaagaaatgcaaaaagaaaatcaaacagctgtttta 1860 $\begin{array}{lllllllllllllllllllll}Q & K & E & I & L & N & L & I & K & E & M & Q & K & E & N & Q & T & A & V & L\end{array}$ tttataagtcatgatt taagtgttatttctgaaatttcagacgatattatagttatgttg 1920

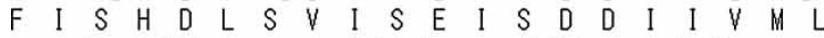
aaaggtaaaatta tagaaaaagcatctactcaacaaattttaaaagatcctaaacaccct 1980

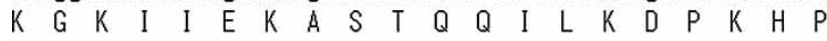
tacactcgcactttat taaataattttttaaaaacaagtattattagttaatattattaa 2040

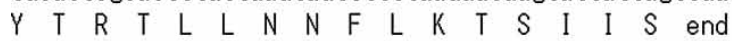

$$
\text { SD }
$$


tttatttgatttattataaaaaaatatt taaaagaaagataaaataaaaatgattttaaa 2100

M I L K

gcaaaaaattattttatatttagctattatattattttcaggtttatcgatttggggaat 2160

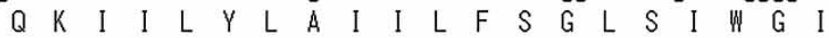

tgttaagtttaaagaatataaagatcatttgtatagaaaagatagaatgattattgctat 2220

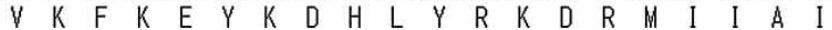

ttcaaattcacctaaaagtttggatttttgtaattcaaaagatactaattctgtttatac 2280

$\begin{array}{lllllllllllllllllll}S & N & S & P & K & S & L & D & F \downarrow C & N & S & K & D & T & N & S & V & Y & T\end{array}$

tgattggactttaggtttatttcatagcactttacttaaggcgccagaaaatcctcaaga 2340

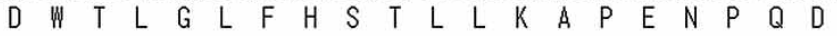

taaacctgaaaacttattagtagaagaatgggatttggacagagagaaccgaaaaataaa 2400

K P E N L L V E E

agccaaattaaaagatggtattttgtttcataacggaaataaaatgactacagaagatgt 2460

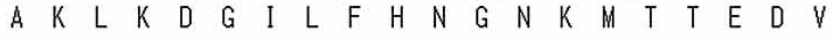

aatttttact tataacagagctgttgaaaaagaacataaagattttgttaacaatataaa 2520

$\begin{array}{lllllllllllllllllllll}I & F & T & Y & N & R & A & V & E & K & E & H & K & D & F & V & N & N & I & K\end{array}$

atctgttcaaaaaatagatgaacttaaatttgaaatccatttaaaagatttacctccatt 2580

$\begin{array}{llllllllllllllllllll}S & V & Q & K & I & D & E & L & K & F & E & I & H & L & K & D & L & P & P & F\end{array}$

ttataattttattttttataaaatgttcagagtcttgaataaagcagctatagacgaaaa 2640

$\begin{array}{llllllllllllllllllll}Y & N & F & I & F & Y & K & M & F & R & V & L & N & K & A & A & I & D & E & N\end{array}$

tgaatcagaaggattaaaaataggaacaggtctt tataaattagtttcggtatcaaaaga 2700

E S E G L K I G T G L Y

taaaaaagat tataatttcgaaaaatttgaaaat tatcatgctaaaaatgattct gaa ta 2760

$\begin{array}{llllllllllllllllllll}K & K & D & Y & N & F & E & K & F & E & N & Y & H & A & K & N & D & S & E & Y\end{array}$

tttagattttgacaaattaccacctaaaataacattaaaagttgatccaagtaacgacaa 2820

L $\quad \begin{array}{lllllllllllllllllll} & \text { F } & D & K & L & P & P & K & \text { I } & T & L & K & V & D & P & S & N & D & N\end{array}$

taatcttttaaacttagaaaaaaagaatatcgatcttattttaagttttccaggtaagaa 2880

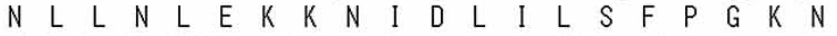

tattaatgataatttaagagataaagaatcaaaaaaaaaattaaacataaaagaggatcg 2940

$\begin{array}{llllllllllllllllllll} & N & D & N & L & R & D & K & E & S & K & K & K & L & N & I & K & E & D & R\end{array}$

acaaacatctaaagttagt tatatgtatataaatcaagaaaacacaaaagaggcaattag 3000

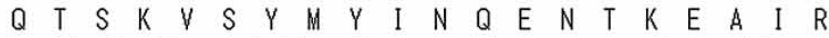

aaaattaatagttcaagctattgatttcgaaaaaataaaagaagaactaaaaattccaaa 3060

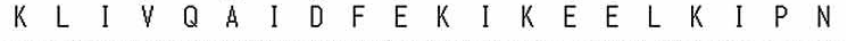

taaagttgctaatggcggtttattacctcctattttaaaaggtcatgataaaacagcaaa 3120

$\begin{array}{llllllllllllllllllll}K & V & \& & N & G & G & L & L & P & P & I & L & K & G & H & D & K & T & A & N\end{array}$

ttatagaatatat tataataaagaattggctaaaacaggtgttcaatcgttaacaggaga 3180

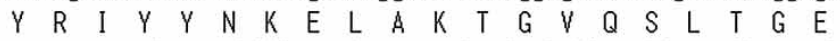

agaaaaaaaataaacattttaacatcagaaaatcctgatcttgcgttgcaattaaaagt 3240

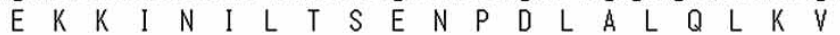

aaaagatcatttagaggaagcaggttttgaagttaaaattaaccaagtacctttcaatga 3300

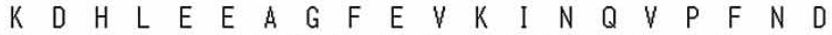

tataacaaacaaattcattactactaat gat tataatattttgtttttaggt gaacaaca 3360

$\begin{array}{llllllllllllllllllll}I & T & N & K & F & I & T & T & N & D & Y & N & I & L & F & L & G & E & Q & H\end{array}$

tgaattgatatatgggtataaattttttgaagat tatttcatacattcagacaaagatcc 3420

$\begin{array}{llllllllllllllllllll}E & L & I & Y & G & Y & K & F & F & E & D & Y & F & I & H & S & D & K & D & P\end{array}$

aaaaaaccaaaatttttcacatataaaagaagaggatgcggacaaaatt tcaaaattaat 3480

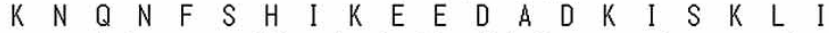

aaaagactctaaggaagctttagatgatgattcatttattcaaaaaataaaagaaataga 3540

$K \begin{array}{lllllllllllllllllll}K & D & S & K & E & A & L & D & D & D & S & F & I & Q & K & I & K & E & I\end{array}$

acaatatttatacacaaaattatacattataccaactttttatgtaact gat tatgtttt 3600

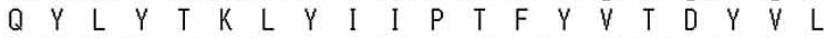

aactagt tctagagtagataaggaacat ttaaaaattaatacatttagt ggt gttaaccc 3660

T S S R V D K E H L K I N

tagatcattaagatttatctcaaaa taaagataatattttataaacttttttgtatattg 3720

R S L R F I S K end

ttataataggacataataataatgcgatatattatcaaaaaaatattttataatttttta 3780

1

attgttttttttatttttttaattagtttttttattttaagaaaaacaactgatccggtt 3840

$\underset{2}{\stackrel{2}{\longrightarrow} 1}$

ttc

2

Figure 2. Nucleotide sequence of the 3903 bp cloned-DNA fragment and deduced amino acid sequences of P36, P30 and P62. The putative -10 and -35 regions of the promoter, and the pyrimidine-rich sequences where the transcription possibly terminates are boldfaced. The Shine-Dalgarno (SD) sequences for each gene and the possible lipoprotein signal peptidase cleavage site are underlined. The vertical arrow indicates the processing site of the P62 precursor protein. The inverted sequences of the $3^{\prime}$-untranslated region are numbered and indicated by arrows. 
1

DPPD_Tpe ----MTEP- -LLRVENLKT YFYTEDG--- ---VVKAVDG VSFEVREGET LGIVGESGSG KSVTSLSIMR LLD-QNGKIV DPPD_Tte ----MARN- -IVEFRNLKT YFYTEEG--- ---VVKAVND VSFSIREGET VCVVGESGCG KSVTALSLMR LIQSPPGKIV OPPD BCl ----MARQN RLLEVENLKT YFHTENG--- ---TVPSVDG VSFHVDRGET VAIVGESGSG KSVTSFSIMG LVS-PPGKIE OPPF_Bsu ---MNELTE KLLEIKHLKQ HFVTPRG--- ---TVKAVDD LSFDIYKGET LGLVGESGCG KSTTGRSIIR LYE-----AT OPPF_BIi -----MAE KLLEIKNLKQ HFSTPKG--- ---IVKAVDG ISFDIYKGET LGLVGESGCG KSTTGRSIIR LYQ-----AT OPPD_Gka -----MARQ KLLEVKNLKQ YFPAGRGQ-- ---LVKAVDG VTFDIYKGET FGLVGESGCG KSTTGRTIIR LYE-----AT DPPF_AYWB527 -MTSSN---Q VLIEIKNLSK NFSIKKNFLK PDTLLKANQN INLSIFKGET LSVVGGSGSG KSTLGQVLLQ LIK-----PT P36 - MLKDRNEKYQ ILIECKNLSK FFFRKKLFAK SSIFLKANDD ISLSIHKGQT LAVVGGSG-- KSTLGQTLLQ LEK------ST DPPD_AYWB528 ------M SLLKVINLHT YFETKKG--- ---LIKAVCG VSFEVQKGKT LGIVGESGSG KSQTAISILK LFE-KNQKIY DPPD_OY192 ------M SLLKVSNLHT YFETQKG--- ---LIKAVRG VSFEVQKGKT LGIVGESGSG KSQTAISILK LFE-KNQKIY P30 -----M KILEVNNLHT YFETEKG--- ---LVKAVQG ISFYLEQGKT LGLVGESGSG KSQTANSILK LFE-KTQKIH

81 GG-EIIFDGR -- ---DILKL SDAEMRRIR- -GNEIGMIFO EPMTSLNPVL TIGDQLMEPL MLHKHMT-KK AG-HIRFDGT --- ----ELTSL SERKMRKVR- -GNEIAMIFQ EPLTSLNPVF TVGHQISEAI LLHQDTK-KA DG-EVLFNGE --- ---NVHGRK SRKKLLEF-- -NRKMQMIFQ DPYASLNPRM TVADIIAEGL DIHKLAKTKK SG-EVLFKGK ------NNHDKK SAKDLLEF-- -NRKMQMIFQ DPYASLNPRM TVADIIAEGI DIHGLAKTKK EG-EVLFNGV ----------NVHGKK SKKELKEL-- -NRKMQMIFQ DPYASLNPRM TVADIIAEGI DIHGLAKTKE SGNVFYYKET TKKAKEFKEK KTKDIEKIDL TTLSNKEKRF LRKDLQIIFQ DPFSSLNTHL KISDIIGEGL LIHKMIKGKE SGKVIYYKEQ --- --- QSINL SELS---- -TKDLQIIFQ NPYLSLNPKI LISDIIGEGL LIHNLVKSKN 8 QG-EITFENR -------- --IISQF SEKEMQKIR- -GNEIAMIFQ DAISSLNPVF KIKNQIIEVL MLHKKLD-YD QG-EITFEKR ---------IISQF SEKEMQKIR- -GNEIAMIFQ DAISSLNPVF KIKNQIIEVL MLHQKLD-YT QG-EIIFNNQ ---------VISDF NEKKMQKIR- -GKEISMIFQ DPITSLNPMF KIKDQIMEIL FIHQEIT-YK

161

200

240

DPPD TPe EARKMAIDLL RKVGIPEPEK RVDEYPHQLS GGMRQRAMIA MALSCRPSLL IADEPTTALD VTIQAQILEL MKELQKEYGM DppD Tte OPPD_BC1 OPPF_Bsu OPPF_B1i OPPD_Gka DPPF_AYWB527 P36 EAWNKAIELI KQVGIPRAEQ IMTSYPHELS GGMRQRIMIA MAISCDPKLL IADEPTTALD VTIQAQILDL LRRLKEEKKM EARKQGIAML KRVGIPRAEQ VYQSYPHALS GGMRQRVMIA MALSCNPKML IADEPTTALD VTIQAQILRL LKKISKEVDT ERMQRVHELL ETVGLN--KE HANRYPHEFS GGQRQRIGIA RALAVDPEFI IADEPISALD VSIQAQVVNL MKELQKEKGL ERLERVHELL NLVGLN--KE HANRYPHEFS GGQRQRIGIA RALAVEPEFI IADEPISALD VSIQAQVVNL MKDLQKERGL ERMQRVYELL ETVGLN--RE HANRYPHEFS GGQRQRIGIA RALAVEPEFI IADEPISALD VSIQAQVVNL LKRLQREKGL DPKYQKMILD IMKKCGIDNY LYDCYPHQLS GGQRQRISIA RALIIKPKFV VCDEIVSALD VSIQSQILNL LNDLKKDYQI DPLYKQKILD IMKKCGVKEE LYNRSLAQLS GGERQRIAIA RTLIIEPKFI VCDEIVSLLD VTIQKQILEL LKQLKKNYEI DPPD_AYWB528 QAYKKTLDIL EKVQIPNAQR VMNSYPHQLS GGMCQRIMIA MALVCKPKLL IADEATTALD VIVQKEILNL IACLQQEQNT DPPD_OY192 QAYQKTLDIL EKVQIPNAQR VMNSYPHQLS GGMCQRIMIA MALVCKPKLL IADEATTALD VIVQKEILNL IACLQQEQNT P30 - EAYEKALNML KKTKIHNCEQ IMNCYPYQLS GGMCQRVMIA IALICQPKLL IVDEATIALD VIVQKEILNL IKEMQKENQT EAYEKALNML KKTKIHNCEQ IMNCYPYQLS GGMCQRVMIA IALICQPKLL IVDEAT $\frac{\text { Walker B }}{\text { C motif }}$

241 280 320

DPPD TPe AIILITHDMG VVAEMSDKVA VMYAGKVVEY GDVKTIFTEP KHPYTYALLE SIPRIDVEQE RLKS-IPGNV PDPLNFPPGC DPPD_Tte ALMLITHDLG IVAEMADYVV VMYAGKVIEE APVRELFKNP KHPYTRGLLK AKPVIGRRQE RLYT-IPGQV PNPIDLGDFC OppD BCl OPPF_Bsu OPPF-B1i OPPD_Gka DPPF_AYWB527 P36 SIILITHDLG VVAELVDRVI VMYAGQIVEQ ADVYTIFKDP KHPYTQGLLE STPKIHELHD ELKS-IRGVV PVPTNMPTGC TYLFIAHDLS MVKYISDRIG VMYFGKLVEL APADELYENP LHPYTKSLLS AIPLPDPDYE RNRVRQKYD- PSVHQLKDGE TYLFIAHDLS MVKYISDRIG VMYFGKLVEL APADELYENP LHPYTQSLLS AIPLPDPDYE RTRVRKTYD- PSVHQLKGDE TYLFIAHDLS MVKYISDRIG VMYFGKMVEL AESEELYRNP IHPYTKALLS AIPLPDPETE RTRKRIVYD- PAQHGYKDGE TFLFITHDLG VARFLSDRIC VMHLGKVIEI APSESIFKNP YHPYTKQIIN AIPKLKTQNK VLEEIPITYE TKKFRFLFQT TLLFITHDLG VAKYLSDQIA IMYLGKLVEL GPTEKIFTNP QHPYTIELLN SIPKLIEYGI SSKKYIAKYE NSSYDFLYNK DPPD_AYWB528 SVLFITHDLG VVSQVADDII VMYRGKIVES AKTSQILKNP QHTYTKSLLN NFLKTGLGYH NF---DPPD OY192 AVLFITHDLG VVSOVADDII VMYQGQIVES AKTSOILKNP KHPYTKSLLN NFLKTGLGYH NV-P30 AVLFISHDIS

\section{H motif}

321

360

\begin{tabular}{|c|c|c|c|c|c|}
\hline PD_Tpe & HPRCEFFE & KGKCDVEEPE & & & \\
\hline PpD_Tte & YFSDRCEYTM & D-VCRKKMPP & LVADENGHKV & ACWLYEG--- & ----EWKK-- \\
\hline $\mathrm{PpD} B \mathrm{BCl}$ & RFHPRCPHAM & D-ICKEKEPM & MVDRDGKAQV & RCWLYAS--- & ----EKEDVG \\
\hline ppF_Bsu & AEFREVKPG & HFVMCTE-AE & FKAFS----- & & \\
\hline PpF_Bli & KPG & -EE & 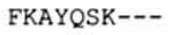 & & 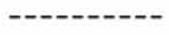 \\
\hline PD_Gka & & & & -1 & ----- \\
\hline & & & & --- & ------ \\
\hline & QQV & & --- & ----- & -------- \\
\hline & -- & & ------ & --- & ----1 \\
\hline & -- & & & & - \\
\hline & ---- & 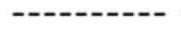 & & -1 & 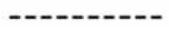 \\
\hline
\end{tabular}

Figure 3. Comparison of P36, P30 and ATP-binding proteins of other microorganisms' ABC transporter systems. The sequences of Walker A, C motif, Walker B and $\mathrm{H}$ motif are underlined and indicated. DppD_Tpe $=$ DppD of Thermotoga petrophila (ZP_01652599); $\mathrm{DppD} \_\mathrm{Tte}=\mathrm{DppD}$ of Thermoanaerobacter tengcongensis (NP_624050); OppD_Bcl = OppD of Bacillus clausii (YP_177152); OppF_Bsu $=\mathrm{OppF}$ of B. subtilis (P24137); OppF_Bli = OppF of Bacillus licheniformis (YP_078436); OppD_Gka = OppD of Geobacillus kaustophilus (YP_146668); DppF_AYWB527 = DppF of aster yellows witches'-broom phytoplasma (YP_456723); DppD_AYWB528 = DppD of aster yellows witches'-broom phytoplasma (YP_456724); DppD_OY192 = DppD of onion yellows phytoplasma (NP_950444). 

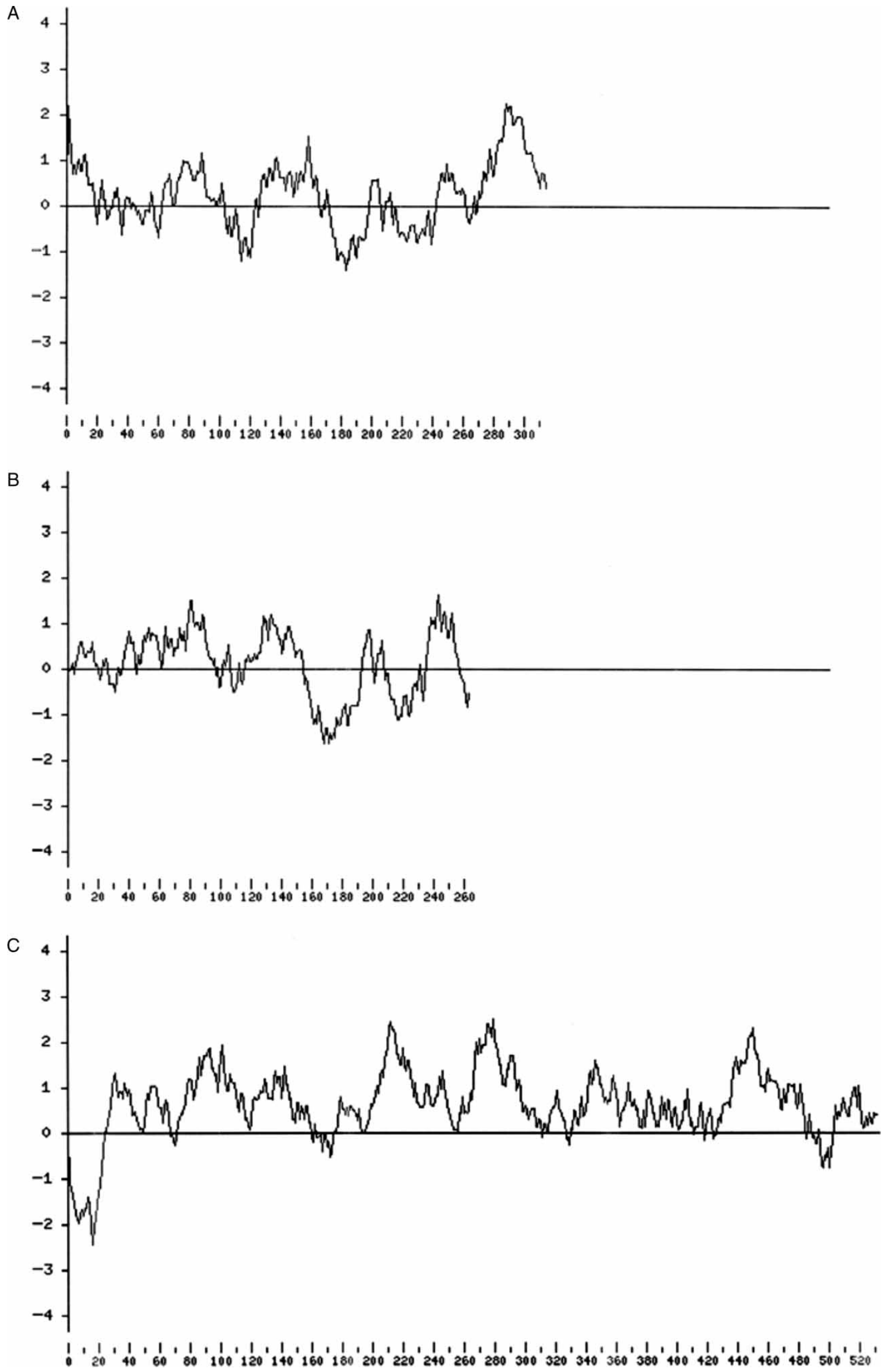

Figure 4. Hydropathy plots of the polypeptides P36 (A), P30 (B) and P62 (C). The hydropathy profiles are obtained according to the method of Kyte and Doolittle (1982), using a window length of 19. Hydrophilic regions are above the line and hydrophobic regions are below. 
are peptide- and nickel-binding proteins that the average sequence similarity score is 0.52 and the identity is low (Tam and Saier 1993). The lipoprotein is the key to understanding the function of the $\mathrm{ABC}$ transporter system. However lipoproteins seldom show homology between each other. It is hard to determine function from comparison of the protein sequences (Tam and Saier 1993). The extracellular solute-binding receptors of bacteria were grouped into eight clusters based on their sequence similarity, and these groupings were generally found to correlate with the molecular sizes and solute-binding specificities of the proteins. The proteins of cluster 5 are the largest of the solute-binding proteins with a size range of 493543 residues. They are more than 100 residues larger than any of the other binding proteins (Tam and Saier 1993). The molecular size of P62 was in the range of the cluster 5 binding proteins.

Since moderate amino acid sequence similarity to ABC-type dipeptide transporter protein, P62 was further submitted to the 3D-PSSM fold recognition server for prediction of secondary structure and then aligned to secondary structure elements of proteins with a solved crystal structure. The secondary structure of P62 showed the most similarity to the $\mathrm{ABC}$ oligopeptide-binding protein AppA of B. subtilis (Levdikov et al. 2005) with an E-value of 1.4e-41 and $100 \%$ estimated precision. AppA is a lipid-modified, membrane-anchored extracellular binding-protein that serves as the receptor for the transport system. It plays important roles in the signal pathway leading to the development of competence and sporulation (Levdikov et al. 2005).

The hydropathy analysis revealed that P62 was a hydrophilic protein with an $\mathrm{N}$-terminal hydrophobic signal peptide and had no obvious membrane spanning domains (Figure 4C). Because the phytoplasma has no periplasmic space, there should be some mechanism to anchor hydrophilic solutebinding proteins to the membrane. There are three types of export sequence of membrane proteins: (1) non-cleavable, membrane-spanning sequence; (2) signal peptides with signal peptidase (SPase) I-like cleavage sites; and (3) signal peptides with SPase IIlike lipoprotein-cleavage sites. There is no strict consensus sequence in the mycoplasma lipoprotein cleavage site besides a Cys in the +1 position. Leu is common at the -3 position in other eubacteria, but not in mycoplasmas (Cleavinger et al. 1995; Sutcliffe and Russell 1995). The region of the putative cleavage site for signal peptide (Figure 2) in P62 had a sequence L-D-F-C-S-N which was consistent with the consensus sequence ( $\mathrm{L}-Y-Z$-cleavage site-C- $y-z$ ) of the lipoprotein precursors (Gilson et al. 1988; Sutcliffe and Russell 1995). In these proteins the $\mathrm{N}$-terminal cysteine is modified into a lipo amino acid that is thought to anchor them to the membrane

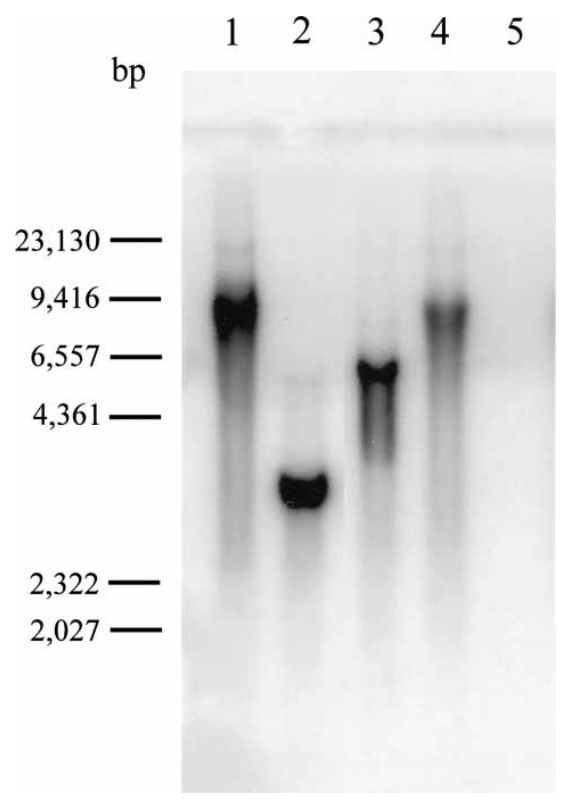

Figure 5. Southern blot analysis of phytoplasma and healthy periwinkle DNA. Lanes $1-4$ are the phytoplasma DNA ( $3 \mu \mathrm{g} / \mathrm{lane})$ digested with BamHI, EcoRI, Pst I and SalI, respectively. Lane 5 is the healthy periwinkle DNA $(10 \mu \mathrm{g})$ digested with $E c o$ RI. The probe used was a ${ }^{32} \mathrm{P}$-labeled RN-P fragment.

(Nielsen and Lampen 1982). These analyses suggested that P62 was a solute-binding lipoprotein specific for peptides and attached to the membrane via the modified $\mathrm{N}$-terminal as in gram-positive bacteria.

\section{Southern blot analysis}

In an attempt to find out the copy number of the $A B C$ transporter system genes, phytoplasma genomic DNA digested with each of the following restriction enzyme: BamHI, EcoRI, Pst I and SalI, and analyzed with a Southern blot probed by a ${ }^{32} \mathrm{P}$-labeled RN-P fragment. There was single band in the individual lanes containing phytoplasma DNA but not in the lane of the healthy periwinkle DNA digested with EcoRI, suggesting that there was only a single copy of the genes in the phytoplasma genome and the genes were LfWB phytoplasma-, but not host- specific (Figure 5).

Phytoplasmas are obligatory parasites in the phloem of host plants, and have a small genome. The complete genome of Candidatus phytoplasma encodes even fewer metabolic functions than that of mycoplasma genomes. The loss of some biosynthetic pathways during the course of evolution results in their inability to be cultured in vitro. They must take up their basic nutrients from the environment through transporter systems to survive. The genomes of OY and AYWB phytoplasmas, and Spiroplasma kunkelii have many genes encoding transporter systems (Oshima et al. 2004; Zhao et al. 2004; Bai et al. 2006). The ABC transport systems of some bacteria are connected with virulence and pathogenesis in the host (Dudler et al. 
1988; Parra-Lopez et al. 1993; Urban et al. 1999; Brown et al. 2001; Liu et al. 2001; Espinasse et al. 2002; Fleissner et al. 2002; Janulczyk et al. 2003). The role of lipoproteins in nutrient transport, cytoadhesion and antigenic variation had been reported in mycoplasmas (Cleavinger et al. 1995; Sutcliffe and Russell 1995). The consumption of metabolites with phytoplasma infection greatly disturbs the metabolic balance of the host cell, and might be a major factor causing disease symptoms (Bai et al. 2006).

In conclusion, we report in this communication an operon isolated from LfWB phytoplasma. The operon contains three structural genes encoding three proteins constructing an $\mathrm{ABC}$ transporter which shares a common $\mathrm{ABC}$ structure and might have the same mechanism of translocation of specific substrates. Southern blot analysis indicated that this ABC transporter system was LfWB phytoplasma-, but not host- specific. It could be a target for designing therapeutic agents against the pathogen.

\section{Acknowledgements}

We thank Dr Te-Chang Lee, Researcher of the Institute of Biomedical Sciences, Academia Sinica, Taiwan for providing aid in DNA autosequencing. Thanks also go to Dr Michael Conrad of University of North Carolina at Chapel Hill for his critical reading and suggestions. This work was financially supported by the National Science Council, Taiwan to K.-C. Ho (Grant No. NSC93-2621-B-002-011).

\section{References}

Ames GFL. 1986. Bacterical periplasmic transport systems: structure, mechanism and evolution. Ann Rev Biochem 55:397-425.

Ankenbauer RG, Nester EW. 1990. Sugar-mediated induction of Agrobacterium tumefaciens virulence genes: Structural specificity and activities of monosaccharides. J Bacteriol 172:6442-6446.

Bai X, Zhang J, Ewing A, Miller SA, Jancso Radek A, Shevchenko DV, Tsukerman K, Walunas T, Lapidus A, Campbell JW, Hogenhout SA. 2006. Living with genome instability: The adaptation of phytoplasmas to diverse environments of their insect and plant hosts. J Bacteriol 188:3682-3696.

Brown JS, Gilliland SM, Holden DW. 2001. A Streptococcus pneumoniae pathogenicity island encoding an $\mathrm{ABC}$ transporter involved in iron uptake and virulence. Mol Microbiol 40:572-585.

Cangelosi GA, Ankenbauer RG, Nester EW. 1990. Sugars induce the Agrobacterium virulence genes through a periplasmic binding protein and a transmembrane signal protein. Proc Natl Acad Sci USA $87: 6708-6712$.

Chen LM, Ho KC. 1997. Characterization of a phytoplasma-related ribonuclease in Catharanthus roseus (L.) G. Don. Taiwania 42:8-12.

Cleavinger CM, Kim MF, Im JH, Wise KS. 1995. Identification of mycoplasma membrane protein by systematic Tn phoA mutagenesis of a recombinant library. Mol Microbiol 18:283-293.
Davies TGE, Coleman JOD. 2000. The Arabidopsis thaliana ATPbinding cassette proteins: An emerging superfamily. Plant Cell Environ 23:431-443.

Dudler R, Schmidhauser C, Parish RW, Wettenhall REH, Schmidt T. 1988. A mycoplasma high-affinity transport system and the in vitro invasiveness of mouse sarcoma cell. EMBO J 7:3963-3970.

Espinasse S, Gohar M, Lereclus D, Sanchis V. 2002. An ABC transporter from Bacillus thuringiensis is essential for betaexotoxin I production. J Bacteriol 184:5848-5854.

Fleissner A, Sopalla C, Weltring KM. 2002. An ATP-binding cassette multidrug-resistance transporter is necessary for tolerance of Gibberella pulicaris to phytoalexins and virulence on potato tubers. Mol Plant Microbe Interact 15:102-108.

Fraser CM, Gocayne JD, White O, Adams MD, Clayton RA, Fleischmann RD, Bult CJ, Kerlavage AR, Sutton G, Kelley JM, Frichman JL, Weidman JF, Small KV, Sandusky M, Fuhrmann J, Nguyen D, Utterback TR, Saudek DM, Phillips CA, Merrick JM, Tomb JF, Dougherty BA, Bott KF, Hu PC, Lucier TS, Peterson SN, Smith HO, Hutchison CA, III, Venter JC. 1995. The minimal gene complement of Mycoplasma genitalium. Science 270:397-403.

Gilson E, Alloing G, Schmidt T, Claverys JP, Dudler R, Hofnung M. 1988. Evidence for high affinity binding-protein dependent transport systems in gram-positive bacteria and in Mycoplasma. EMBO J 7:3971-3974.

Gundersen DE, Lee IM, Rehner SA, Davis RE, Kingsbury DT. 1994. Phylogeny of mycoplasmalike organisms (phytoplasmas): A basis for their classification. J Bacteriol 176:5244-5254.

Higgins CF. 1992. ABC transporters: From microorganism to man. Ann Rev Cell Biol 8:67-113.

Ho KC, Tsai CC, Chung TL. 2001. Organization of ribosomal RNA genes from a loofah witches' broom phytoplasma. DNA Cell Biol 20:115-122.

Janulczyk R, Ricci S, Bjorck L. 2003. MtsABC is important for manganese and iron transport, oxidative stress resistance, and virulence of Streptococcus pyogenes. Infect Immun 71:2656-2664.

Kelley LA, MacCallum RM, Sternberg MJ. 2000. Enhanced genome annotation using structural profiles in the program $3 \mathrm{~d}-$ pssm. J Mol Biol 299:499-520.

Khwaja M, Ma Q, Saier MH, Jr.. 2005. Topological analysis of integral membrane constituents of prokaryotic ABC efflux systems. Res Microbiol 156:270-277.

Kirkpatrick BC. 1989. Strategies for characterizing plant pathogenic mycoplasma-like organisms and their effects on plants. In: Kosuge T, Nester E, editors. Plant-microbe interactions, molecular and genetic perspectives. Vol 3. New York: McGraw-Hill Publishing Co. p 241-293.

Kirkpatrick BC, Smart CD. 1995. Phytoplasmas: Can phylogeny provide the means to understand pathogenicity. Adv Bot Res $21: 187-212$.

Koide A, Hoch JA. 1994. Identification of a second oligopeptide transport system in Bacillus subtilis and determination of its role in sporulation. Mol Microbiol 13:417-426.

Kyte J, Doolittle RF. 1982. A simple method for displaying the hydropathic character of a protein. J Mol Biol 157:105-132.

Lee IM, Davis RE. 1986. Prospects for in vitro culture of plantpathogenic mycoplasma-like organisms. Ann Rev Phytopathol 24:339-354.

Lee IM, Davis RE, Gundersen-Rindal DE. 2000. Phytoplasma: Phytopathogenic mollicutes. Ann Rev Microbiol 54:221-255.

Levdikov VM, Blagova EV, Brannigan JA, Wright L, Vagin AA, Wilkinson AJ. 2005. The structure of the oligopeptide-binding protein, AppA, from Bacillus subtilis in complex with a nonapeptide. J Mol Biol 345:879-892.

Liu Z, Jacobs M, Schaff DA, McCullen CA, Binns AN. 2001. ChvD, a chromosomally encoded ATP-binding cassette transporter-homologous protein involved in regulation of virulence gene expression in Agrobacterium tumefaciens. J Bacteriol 183:3310-3317. 
Locher KP. 2004. Structure and mechanism of ABC tansporters. Curr Opin Struct Biol 14:426-431.

McCoy RE, Caudwell A, Chang CJ, Chen TA, Chiykowski LN, Gousins NT, Dale JL, Leeuw GTN, Golino DA, Hackett KJ, Kirkpatrick BC, Marwitz R, Petzold H, Sinha RC, Sugiura M, Whitcomb RF, Yang IL, Zhu BM, Seemuller E. 1989. Plant diseases associated with mycoplasma-like organisms. In: Whitcomb RF, Tully JG, editors. The mycoplasmas. Vol. 5. New York: Academic Press. p 545-640.

McGrath JP, Varshavsky A. 1989. The Yeast STE6 gene encodes a homologue of the mammalian multidrug resistance P-glycoprotein. Nature 340:400-404.

Monnet V. 2003. Bacterial oligopeptide-binding proteins. Cell Mol Life Sci 60:2100-2114.

Nielsen JBK, Lampen JO. 1982. Glyceride-cycteine lipoproteins and secretion by gram-positive bacteria. J Bacteriol 152:315-322.

Oshima K, Kakizawa S, Nishigawa H, Jung HY, Wei W, Suzuki S, Arashida R, Nakata D, Miyata S, Ugaki M, Namba S. 2004. Reductive evolution suggested from the complete genome sequence of a plant-pathogenic phytoplasma. Nat Genet $36: 27-29$.

Parra-Lopez C, Baer M, Groisman EA. 1993. Molecular genetic analysis of a locus required of resistance to antimicrobial peptides in Salmonella typhimurium. EMBO J 12:4053-4062.

Perego M, Higgins CF, Pearce SR, Gallagher MP, Hoch JA. 1991. The oligopeptide transport system of Bacillus subtilis plays a role in the initiation of sporulation. Mol Microbiol 5:137-185.

Razin S. 1992. Mycoplasma taxonomy and ecology. In: Maniloff J, McElhaney RN, Finch LR, Baseman J, editors. Mycoplasma: Molecular biology and pathogenesis. Washington DC: ASM. p 3-22.

Riordan JR, Rommens JM, Kerem BS, Alon N, Rozmahel R, Grzelczak J, Zielenski J, Lok S, Plavsk N, Chou JL, Drumm ML, Ianuzzi FS, Collins FS, Tsui LC. 1989. Identification of the cystic fibrosis gene: Cloning and characterization of complementary DNA. Science 245:1066-1073.

Saier MH, Jr. 1993. Protein phosphorylation and signal transduction in bacteria: An introduction. J Cell Biochem 51:61-68.
Sears BB, Kirkpatrick BC. 1994. Unveiling the evolutionary relationship of plant pathogenic mycoplasma-like organisms: Phylogenetic insights may provide the key to culturing, phytoplasma. ASM News 60:307-312.

Shuman HA. 1987. The genetics of active transport in bacteria. Ann Rev Genet 21:155-177.

Sutcliffe IC, Russell RRB. 1995. Lipoproteins of gram-positive bacteria. J Bacteriol 177:1123-1128.

Tam R, Saier MH, Jr. 1993. Structural, functional, and evolutionary relationship among extracellullar solute-binding receptor of bacteria. Microbiol Rev 57:320-346.

Tata F, Stanier P, Wicking C, Halford S, Kruyer H, Lench NJ, Scambler PJ, Hanse C, Braman JC, Williamson R, Wainwright BJ. 1991. Cloning the mouse homologue of the human cystic fibrosis transmembrane conductance regulator gene. Genomics 10:301-307.

Thompson JD, Gibson TJ, Plewniak F, Jeanmougin F, Higgins DG. 1997. The clustaX windows interface: Flexuble strategies for multiple sequence alignment aided by quality analysis tools. Nucl Acids Res 24:4876-4882.

Tully JG. 1993. International committee on systemic bacteriology subcommittee on the taxonomy of millicutes: Minutes of interim meeting. 1-2 August, Ames, Iowa Int J Syst Bacteriol 43:394-397.

Urban M, Bhargava T, Hamer JE. 1999. An ATP-driven efflux pump is a noval pathogenicity factor in rice blast disease. EMBO J 18:512-521.

Wagner M, Fingerhut C, Gross HJ, Schön A. 2001. The first phytoplasma RNase P RNA provides new insights into the sequence requirements of this ribozyme. Nucl Acids Res 29:2661-2665.

Young L, Leonhard K, Tatsuta T, Trowsdale J, Langer T. 2001. Role of the ABC transporter Mdl1 in peptide export from mitochondria. Science 291:2135-2138.

Zhao Y, Wang H, Hammond RW, Jomantiene R, Liu Q, Lin S, Roe BA, Davis RE. 2004. Predicted ATP-binding cassette systems in the phytopathogenic mollicute Spiroplasma kunkelii. Mol Gen Genomics 271:325-338. 